\title{
Makeup of the genetic correlation between milk production traits using genome-wide single nucleotide polymorphism information
}

\author{
R. van Binsbergen, ${ }^{\star} \dagger$ R. F. Veerkamp, $\dagger^{1}$ and M. P. L. Calus $\dagger$ \\ ${ }^{*}$ Animal Breeding and Genomics Centre, Wageningen University, PO Box 338, $6700 \mathrm{AH}$ Wageningen, the Netherlands \\ †Animal Breeding and Genomics Centre, Wageningen UR Livestock Research, PO Box 65, 8200 AB Lelystad, the Netherlands
}

\begin{abstract}
The correlated responses between traits may differ depending on the makeup of genetic covariances, and may differ from the predictions of polygenic covariances Therefore, the objective of the present study was to investigate the makeup of the genetic covariances between the well-studied traits: milk yield, fat yield, protein yield, and their percentages in more detail. Phenotypic records of 1,737 heifers of research farms in 4 different countries were used after homogenizing and adjusting for management effects. All cows had a genotype for 37,590 single nucleotide polymorphisms (SNP). A Bayesian stochastic search variable selection model was used to estimate the SNP effects for each trait. About 0.5 to $1.0 \%$ of the SNP had a significant effect on 1 or more traits; however, the SNP without a significant effect explained most of the genetic variances and covariances of the traits. Single nucleotide polymorphism correlations differed from the polygenic correlations, but only 10 regions were found with an effect on multiple traits; in 1 of these regions the DGAT1 gene was previously reported with an effect on multiple traits. This region explained up to $41 \%$ of the variances of 4 traits and explained a major part of the correlation between fat yield and fat percentage and contributes to asymmetry in correlated response between fat yield and fat percentage. Overall, for the traits in this study, the infinitesimal model is expected to be sufficient for the estimation of the variances and covariances.
\end{abstract}

Key words: genome-wide association, correlation, covariance, dairy cow

\section{INTRODUCTION}

Genetic association between traits and correlated responses resulting from those associations are generally predicted using genetic correlations; for example, between milk production (Visscher and Thompson, 1992) and health (for review, see Rauw et al., 1998)

Received July 13, 2011.

Accepted December 27, 2011

${ }^{1}$ Corresponding author: Roel.Veerkamp@wur.nl or fertility (for review, see Veerkamp et al., 2003) in dairy cows. Using the genetic correlation, however, has limitations due to the assumption of the underlying infinitesimal model. Genetic correlations do not give insight in some aspects of the underlying biology, such as, for example, the causality of the genetic effect of high yield on fertility, or vice versa. This is because genetic associations between traits are assumed to be linear and symmetric (e.g., Bohren et al., 1966). Also, genetic correlations will be affected by selection for one (Parker et al., 1970) or both of the traits, depending on the genetic makeup of the covariances (McMillan et al., 1995; Quinton and McMillan, 1996). To evaluate the makeup of the covariance between yield and percentage traits, Lipkin et al. (2008a) and Visscher and Thompson (1992) modeled the genetic covariance between the yield and percentage traits, starting from the QTL variances and polygenic components, respectively. Using simulation studies and deriving empirical equations, Bohren et al. (1966) concluded that asymmetry of correlated responses depends on the gene actions that underlie the genetic covariance and that genetic covariances are likely to be more sensitive to selection than variances (i.e., the allele substitution effects and allele frequencies might change after several generations). For these reasons, if the makeup of the genetic covariance differs from the assumptions of the infinitesimal model, this has consequences for the longterm selection responses. However, the consequences are broader; for example, most current applications of genomic prediction are single trait, and in a second step the genomic predictions of individual traits are combined with genomic or polygenic EBV from other or the same traits across many generations. Combining these different predictions, however, requires making assumptions on the makeup of the genetic covariance. If the makeup of the covariance differs from the polygenic infinitesimal assumptions and changes over time due to selection, multiple-trait genomic selection procedures (e.g., Calus and Veerkamp, 2011) are required to use high-density SNP genotypes to predict across traits accurately following changes in allele frequencies over time. 
Several QTL studies, using either within-family linkage information or genome-wide association studies, investigated the effects of individual QTL on one (for review, see Khatkar et al., 2004) or more milk production traits (Schrooten et al., 2004; Bagnato et al., 2008; Chamberlain et al., 2008; Lipkin et al., 2008b; Bolormaa et al., 2010). However, even based on the QTL of genome-wide association studies, it is not easy to investigate the makeup of the genetic (co)variances between traits. Quantitative trait loci are often not very precisely located and the significant SNP are likely to be the ones in highest linkage disequilibrium rather than the closest location on the genome. Also, multiple testing hinders the dissecting of the covariance between traits into causative QTL. One way to overcome these testing problems is to use a validation population in a different population or breed (Bagnato et al., 2008; Bolormaa et al., 2010), assume a relatively low type II error (Lipkin et al., 2008b), or use a low type I error but corrected for a high type II error (Chamberlain et al., 2008). The studies reported by Bagnato et al. (2008) and Lipkin et al. (2008b) reported that half of the QTL affected both milk yield and protein percentage. The study by Chamberlain et al. (2008) suggested that most QTL affected one trait and not the other milk production traits. Other methods might include adjusting one trait for the other trait in the model finding QTL, or using principle component analysis (Bolormaa et al., 2010). However, it is still difficult to determine the QTL that makeup the genetic (co)variances between traits, especially because individual QTL explain little variance across the population, although sometimes within-family linkage studies do reveal large putative QTL. Comparing published genetic correlations to correlation estimates based on reported QTL effects for different QTL mapping populations generally showed a poor match between both estimates (Gardner and Latta, 2007) .

With denser marker genotypes for dairy cattle, genome-wide association studies are likely to pick up more of the (co)variance for traits. Therefore, using SNP estimates across the genome might be more useful to investigate the total makeup of the genetic covariance between 2 traits in a population, rather than focusing on the mapped QTL that explain relatively little of the total genetic variances. Bolormaa et al. (2010) estimated SNP solutions of milk yield, fat yield, protein yield, and their percentages for Holstein-Friesian cows. They used SNP-by-SNP analysis, where each SNP was fitted individually. One drawback from such analysis is that estimated effects of individual SNP tend to be overestimated, which, in turn, may affect the estimated genetic correlation and its makeup. The objective of the present study was to investigate the makeup of the genetic covariances between milk production traits, focusing on the total genetic covariance explained, rather than identifying individual QTL, using a Bayesian stochastic search variable selection (BSSVS) model to estimate all SNP effects simultaneously.

\section{MATERIALS AND METHODS}

The data used in the present study originated from Teagasc Moorepark Dairy Production Research Center (Ireland), Scottish Agricultural College (Edinburgh, UK), Wageningen UR Livestock Research (Lelystad, the Netherlands), and The Swedish University of Agricultural Science (Uppsala, Sweden).

\section{Phenotypic Data}

Phenotypic data were available of 1,804 first-lactation Holstein heifers with 66,116 test-day records of 45 wk for milk, fat, and protein yields. Because the data came from different herds with different management and different frequencies of data recording, phenotypic data were preadjusted for management effects. For this purpose, a single-trait test-day model was fitted with a random animal effect as a sixth-order polynomial (no genetic relationships included), a fixed effect for the mean lactation curve (fourth-order polynomial), and a deviation of this mean curve for 10 management groups formed by farm, nutritional system, and milking frequency combinations (third-order polynomial). Specific time-dependent random effects were fitted for yearmonth of test by management group (353 levels) and a specific treatment effect was fitted for experimental treatments during lactation for the cows in Ireland (81 levels). The model was fitted in ASReml (Gilmour et al., 2009) and used to predict for each cow a full lactation curve, using the overall mean and the animal effects only. The averages of the predicted animal effects for wk 3 to 15 were used in the association study for all animals with more than 10 observations in this period in the data set. Average fat and protein percentages were calculated from the average milk yield and the average fat and protein yields, respectively.

\section{Genotypic Data}

All animals with phenotypic information were genotyped with the Illumina BovineSNP50 BeadChip (Illumina Inc., San Diego, CA) containing 54,001 SNP. Single nucleotide polymorphisms that fulfilled the following criteria were included in the association study: 1) gene calling (GC) score $>0.20$ and genetic testing (GT) score $>0.55 ; 2$ ) call rate $>95 \%$; 3 ) minor allele frequency $>0.01$ in each country; and 4) no extreme 
deviation from Hardy-Weinberg equilibrium (i.e., $\chi^{2}$ $<600)$. The GC score and GT score are quality measures on the genotype calls from the genotyping assay. After the quality control, 37,590 SNP remained. Checks for Mendelian inconsistencies between pedigree and SNP data were performed for all genotyped parent-offspring pairs and among sibs (Calus et al., 2011a). After these edits, 1,737 animals with phenotypes and genotypes remained in the data. Missing genotypes were imputed using Beagle software (Browning and Browning, 2007). Chromosome number and locations of the SNP on the BovineSNP50 were obtained from Illumina Inc. (March 2011) for the UMD3.0 bovine genome assembly from the University of Maryland (College Park, MD).

\section{Statistical Model}

The single-trait BSSVS model (described by Calus et al., 2008; Verbyla et al., 2010) was used to sample whether SNP were linked to a QTL or not. The model used was

$$
y_{i}=\mu+\operatorname{animal}_{i}+\sum_{j=1}^{n l o c} \sum_{k=1}^{2} S N P_{i j k}+e_{i},
$$

where $y_{i}$ is the phenotypic record of animal $i, \mu$ is the overall mean, animal $i$ is the random polygenic effect of animal $i, n l o c$ is the number of SNP included, $S N P_{i j k}$ is a random effect for allele $k$ at locus $j$ of animal $i$, and $e_{i}$ is a random residual for animal $i$. The vectors animal and e where assumed to be normally distributed animal $\sim \mathbf{N}\left(0, \mathbf{A} \sigma_{u}{ }^{2}\right)$ and $\mathbf{e} \sim \mathbf{N}\left(0, \mathbf{I} \sigma_{e}{ }^{2}\right)$, where $\mathbf{A}$ is the numerator relationship matrix and $\mathbf{I}$ is an identity matrix].

To allow for scenarios where a limited number of genes of large effects exist and for scenarios where a large number of genes all had an infinite small effect (the infinitesimal model), an indicator variable $I_{j}$ was introduced into the hierarchical model. This indicator variable takes a value of 1 or 0 , representing whether the SNP was included with a large effect in the model or not. The QTL indicator $I_{j}$ was sampled from

$$
\begin{gathered}
I_{j} \mid v_{j}, V \sim \text { Bernoulli } \\
{\left[\frac{\varphi\left(v_{j} ; 0, V\right) \times p_{j}}{\varphi\left(v_{j} ; 0, V\right) \times p_{j}+\varphi\left(v_{j} ; 0, \frac{V}{100}\right) \times\left(1-p_{j}\right)}\right],}
\end{gathered}
$$

where $p_{j}$ is the prior QTL probability (i.e., the probability that $I_{j}$ is equal to 1 ), which follows a Bernoulli distribution $\varphi\left(v_{j} ; 0, V\right)$, with mean 0 and variance $V$. Prior
QTL probability used in the analyses had a value of 0.00027 , reflecting the prior assumption that 10 major QTL underlie each of the traits. This is equivalent to a value of 0.99973 for $\pi$. Note that the parameter $\pi$ is commonly used for BayesB-type models to denote the proportions of SNP that are not included in the model and, therefore, have zero effect, whereas for BSSVS, it defines the proportion of loci with effects sampled from the distribution with small effects. The posterior distribution of $I_{j}$ showed the proportion of cycles for which the effects for locus $j$ were sampled from the distribution with large effects. Single nucleotide polymorphisms with the highest posterior probability were expected to be most likely linked to a QTL.

Single nucleotide polymorphism effects, in the model denoted as $S N P_{i j k}$, were estimated as $q_{i j k} \times \mathrm{v}_{j}$ (Meuwissen and Goddard, 2004), where $q_{i j k}$ is the size of the effect of allele $k$ at locus $j$ and $\mathrm{v}_{j}$ is a scaling vector for locus $j$. Both $q_{i j k}$ and $v_{j}$ are estimated in the model. The prior distribution for variance of $\mathrm{v}_{j}$, denoted as $\mathrm{V}$, was (Meuwissen and Goddard, 2004)

$$
\mathrm{p}(\mathrm{V})=\chi^{-2}\left(\mathrm{~S}_{0}, 10\right)
$$

where $\mathrm{S}_{0}$ was chosen such that it reflected the expected variance of a locus with a QTL probability of 1 . This expected variance was calculated as the total genetic variance of the analyzed trait, divided by nloc $\times\left(p_{j}+\frac{1-p_{j}}{100}\right) ; \mathrm{V}$ was sampled from the following conditional inverted $\chi^{2}$ distribution with $(n l o c-1+$ 10) degrees of freedom:

$$
\mathrm{V} \mid \text { v.,I. } \sim \chi^{-2}\left(S_{0}+S Z, n l o c-1+10\right),
$$

where $v$. and $I$. are $v$ and $I$ across all loci and

$$
S Z=\sum_{k=1}^{n l o c} v_{j}^{2}\left[I_{j}+\left(1-I_{j}\right) \times 100\right] .
$$

\section{Analysis}

First, the genetic and residual variances and genetic correlations were estimated between all traits using pedigree relationships in multivariate linear mixed models in ASReml (Gilmour et al., 2009). The estimated variances were used to calculate heritabilities and to infer prior variances for the BSSVS model described above to estimate SNP effects. The BSSVS model was performed using Gibbs sampling; each time the model was run for 50,000 cycles with 10,000 cycles disregarded for burn-in. For the prior QTL variance, it was assumed 
that $80 \%$ of the genetic variance was explained by the SNP and the remaining $20 \%$ by the polygenic component in the model. The SNP variance was split across 10 SNP sampled from a distribution with a large effect and the remaining 37,580 SNP coming from a distribution with small effect (i.e., with an SNP variance that is 100 times smaller than the SNP with a large effect). Each trait was analyzed with 5 different Gibbs chains of 50,000 cycles and average results of the 5 chains were used in the further study.

An SNP was assumed to be significant if the Bayes Factor (BF; Kass and Raftery, 1995) was equal or higher than 3.1, which is termed a substantial effect. The Bayes Factor was calculated for each SNP by

$$
B F=\frac{\operatorname{Pr}\left(H_{1} \mid y\right)}{1-\operatorname{Pr}\left(H_{1} \mid y\right)} \div \frac{\operatorname{Pr}\left(H_{1}\right)}{1-\operatorname{Pr}\left(H_{1}\right)},
$$

where $H_{1}$ is the hypothesis that the marker is linked to a QTL, $\operatorname{Pr}\left(H_{1} \mid y\right)$ is the posterior probability of the hypothesis and $\operatorname{Pr}\left(H_{1}\right)$ is the prior probability of the hypothesis; $1-\operatorname{Pr}\left(H_{1} \mid y\right)$ and $1-\operatorname{Pr}\left(H_{1}\right)$ represent, respectively, the posterior and prior probability for the alternative hypothesis.

Because many SNP are closely linked on the chromosome, it was difficult to differentiate between neighboring SNP that are in close linkage disequilibrium. Because of lack of this differentiating power between SNP, incomplete mixing of the sampler was observed and different sets of SNP gave equivalent good fit to the data in different chains. Typically, in one chain an SNP was fairly quickly fixed as coming from the distribution, reflecting a large effect, whereas in a different chain this happened to a neighboring SNP. To ensure that the average of the chains reflects the importance of the region, the sum of the posterior and prior QTL probabilities of a window of 10 SNP (an SNP plus its closest neighbors) also was used. Use of these summed probabilities might reveal regions with SNP effects that might be missed otherwise, because multiple SNP in a region together explained the same QTL.

\section{SNP Variances, Covariances, and Correlations}

The standardized genetic variance of trait $i$ was calculated for each SNP $k$ separately:

$$
\sigma_{i k}^{\prime 2}=\frac{\sigma_{i k}^{2}}{\sum_{l=1}^{n S N P}\left(\sigma_{i l}^{2}\right)}=\frac{2 p_{k} q_{k} \times a_{i k}^{2}}{\sum_{l=1}^{n S N P}\left(2 p_{l} q_{l} \times a_{i l}^{2}\right)}
$$

where $a_{i k}^{2}$ is the posterior mean of the squared allele substitution effect of SNP $k$ for trait $i, n S N P$ is the number of SNP included, and $p$. and $q$. are the allele frequencies for SNP $a$ and $l$. The sum of the variances of all SNP of trait $i$ gave the total variance explained by the SNP of trait $i$. The standardized genetic covariance between trait $i$ and $j$ per SNP $k\left(\sigma_{i j k}^{\prime}\right)$ was calculated as

$$
\sigma_{i j k}^{\prime}=\frac{2 p_{k} q_{k} \times a_{i k} a_{j k}}{\sum_{l=1}^{n S N P}\left(2 p_{l} q_{l} \times a_{i l} \times a_{j l}\right)} .
$$

The sum of the standardized covariances of all SNP gave the total standardized covariance between 2 traits, which is equal to the correlation as standardized variances were 1 .

Bohren et al. (1966) considered 4 types of loci with different gene effects on 2 traits: type $\mathrm{A}$ and $\mathrm{D}$ affected 1 of the 2 traits; type $\mathrm{B}$ affected both traits in the same direction; and type $\mathrm{C}$ affected both traits but in opposite directions. Type A and D loci are expected to contribute little to the covariance between traits (but affect the correlation through the variance), because they affect a single trait with a large effect and the other trait with a minor effect, whereas type B and C loci influence both traits with a large effect and, therefore, do contribute to the covariance between 2 traits significantly. In the present study, the correlated traits were split up according to these 4 types of loci. To assign a certain SNP to a class, an SNP was assumed to have an effect if its BF was equal to or higher than 3.1. For SNP that did not fit into the 4 classes (i.e., no effect for both traits), a fifth class (class 0) was made. For each locus class, the number of SNP, standardized SNP variances, and standardized SNP covariances were calculated. The next step was to find SNP regions, rather than individual SNP, that were significant for 2 traits. Single nucleotide polymorphism regions where selected by using the sum of the posterior and prior probabilities for a window of 10 SNP (an SNP plus its closest neighbors) for calculation of the BF. The total standardized variance of the traits and the standardized covariance between correlated traits were calculated for each SNP region. Manhattan plots were made to visualize the locations of the significant SNP regions.

\section{RESULTS}

The original data set contained phenotypic data of 1,804 cows. After standardizing and adjusting, 1,737 cows had records for milk yield and 1,629 cows had records for fat and protein yield and their percentages. Summary statistics for the adjusted milk, fat, and pro- 
Table 1. Number of records, mean, phenotypic standard deviation, and range for the production traits after adjustment for management effects

\begin{tabular}{lccccc}
\hline Item & $\mathrm{n}$ & Mean & $\mathrm{SD}$ & Minimum & Maximum \\
\hline Milk (kg) & 1,737 & 24.0 & 4.5 & 1.0 & 39.4 \\
Fat (kg) & 1,629 & 0.93 & 0.18 & 0.12 & 1.80 \\
Protein (kg) & 1,629 & 0.73 & 0.13 & 0.05 & 1.34 \\
Fat (\%) & 1,629 & 3.95 & 0.57 & 2.16 & 12.38 \\
Protein (\%) & 1,629 & 3.06 & 0.27 & 1.62 & 7.25 \\
\hline
\end{tabular}

tein yields and fat and protein percentages are given in Table 1. Polygenic heritability estimates with ASReml ranged from 0.36 for milk yield to 0.54 for fat percentage (Table 2).

The BF for all SNP for the 5 traits are shown in Figure 1. Significant SNP were found on most chromosomes (for example, a region for milk production at the $\mathrm{X}$ chromosome or a region for protein percentage on chromosome 6). Per trait 0.5 to $1.0 \%$ of the SNP had a $\mathrm{BF}$ equal to or greater than 3.1 (i.e., 200, 308 and 334 SNP for protein, fat, and milk yield, and 318 and 387 for fat and protein percentages, respectively). When the $\mathrm{BF}$ was averaged across regions using a sliding window of 10 neighboring SNP, less significant SNP were identified (Figure 2); in total, 16 regions were found for milk yield, 10 for fat yield, 7 for protein yield, 12 for fat percentage, and 15 for protein percentage with a $\mathrm{BF}$ equal or higher than 3.1.

Estimates for the total phenotypic variances were similar using ASReml or using the SNP plus polygenic variances from BSSVS (Table 2). For the percentage traits, relatively more genetic variance was picked up by the BSSVS compared with ASReml and for protein percentage, the SNP explained only half of the genetic variance estimated with BSSVS. The polygenic genetic correlations between milk, protein, and fat yield (Table 3) ranged from 0.57 to 0.85 with ASReml, and the SNP correlations from 0.52 to 0.68 using BSSVS. In general, genetic correlations estimated with BSSVS were lower than genetic correlations estimated with ASReml, ex- cept for the genetic correlation between protein yield and fat percentage. The correlation between fat and protein percentages differed most between both methods (0.72 vs. 0.33) for ASReml and BSSVS, respectively. When the polygenic variance of BSSVS was included in the genetic correlation (i.e., no polygenic covariance was fitted), then the BSSVS genetic correlations were even lower, especially when protein yield was involved, as the polygenic effect explained a relatively large part of the genetic variance (Table 2).

To investigate the makeup of the genetic covariance, significant SNP were divided in the type of loci classes given by Bohren et al. (1966), with an additional class 0 for the SNP with $\mathrm{BF}<3.1$ for both traits. For the correlation between milk yield and fat yield, 37,008 SNP were in class 0; $274 \mathrm{SNP}$ in class A, and $248 \mathrm{SNP}$ in class D. Class B and C contained, respectively, 58 and 2 SNP. This pattern of the most SNP in class 0 and the fewest in class B and C was also observed for the other genetic correlations (Table 4) and, as expected, highly correlated traits had more SNP in class B/C, positively correlated traits had the most SNP in class $\mathrm{B}$, and negatively correlated traits had the most SNP in class C.

The SNP in class 0 explained most of the genetic variance (Table 5; for example, 96-97\% of the SNP variance of milk production). The other traits showed comparable results, except for fat percentage where $55 \%$ was explained by SNP in class 0 and more than $40 \%$ by $\mathrm{SNP}$ in class $\mathrm{B}, \mathrm{C}$, or $\mathrm{D}$, depending on the

Table 2. Polygenic $(\mathrm{G})$, residual $(\mathrm{R})$, phenotypic $(\mathrm{P})$, total SNP variances $(\mathrm{SNP})$, and the heritability $\left(\mathrm{h}^{2}\right)$ for the production traits calculated traditionally (restricted maximum likelihood, REML) and using genome-wide SNP information (Bayesian stochastic search variable selection, BSSVS $)^{1}$

\begin{tabular}{|c|c|c|c|c|c|c|c|c|c|c|}
\hline \multirow[b]{2}{*}{ Trait } & \multicolumn{4}{|c|}{ REML } & \multicolumn{6}{|c|}{ BSSVS } \\
\hline & $\mathrm{G}$ & $\mathrm{R}$ & $\mathrm{P}$ & $\mathrm{h}^{2}$ & SNP & G & $\mathrm{R}$ & $\mathrm{P}$ & $\mathrm{h}_{\mathrm{tot}}^{2}$ & $\mathrm{~h}_{\mathrm{SNP}}^{2}$ \\
\hline Fat (kg) & 0.012 & 0.018 & 0.031 & 0.41 & 0.010 & 0.002 & 0.018 & 0.030 & 0.40 & 0.33 \\
\hline Protein $(\mathrm{kg})$ & 0.006 & 0.010 & 0.016 & 0.38 & 0.003 & 0.003 & 0.010 & 0.016 & 0.38 & 0.19 \\
\hline Fat (\%) & 0.18 & 0.15 & 0.33 & 0.54 & 0.15 & 0.06 & 0.12 & 0.33 & 0.64 & 0.45 \\
\hline Protein $(\%)$ & 0.03 & 0.04 & 0.07 & 0.42 & 0.03 & 0.01 & 0.04 & 0.07 & 0.50 & 0.38 \\
\hline
\end{tabular}

${ }^{1}$ For genome-wide association studies, the genetic variance was split up in SNP variances and polygenic variances; $h_{\text {tot }}^{2}$ includes both genetic variances, whereas $\mathrm{h}_{\mathrm{SNP}}^{2}$ includes the SNP variance only. 


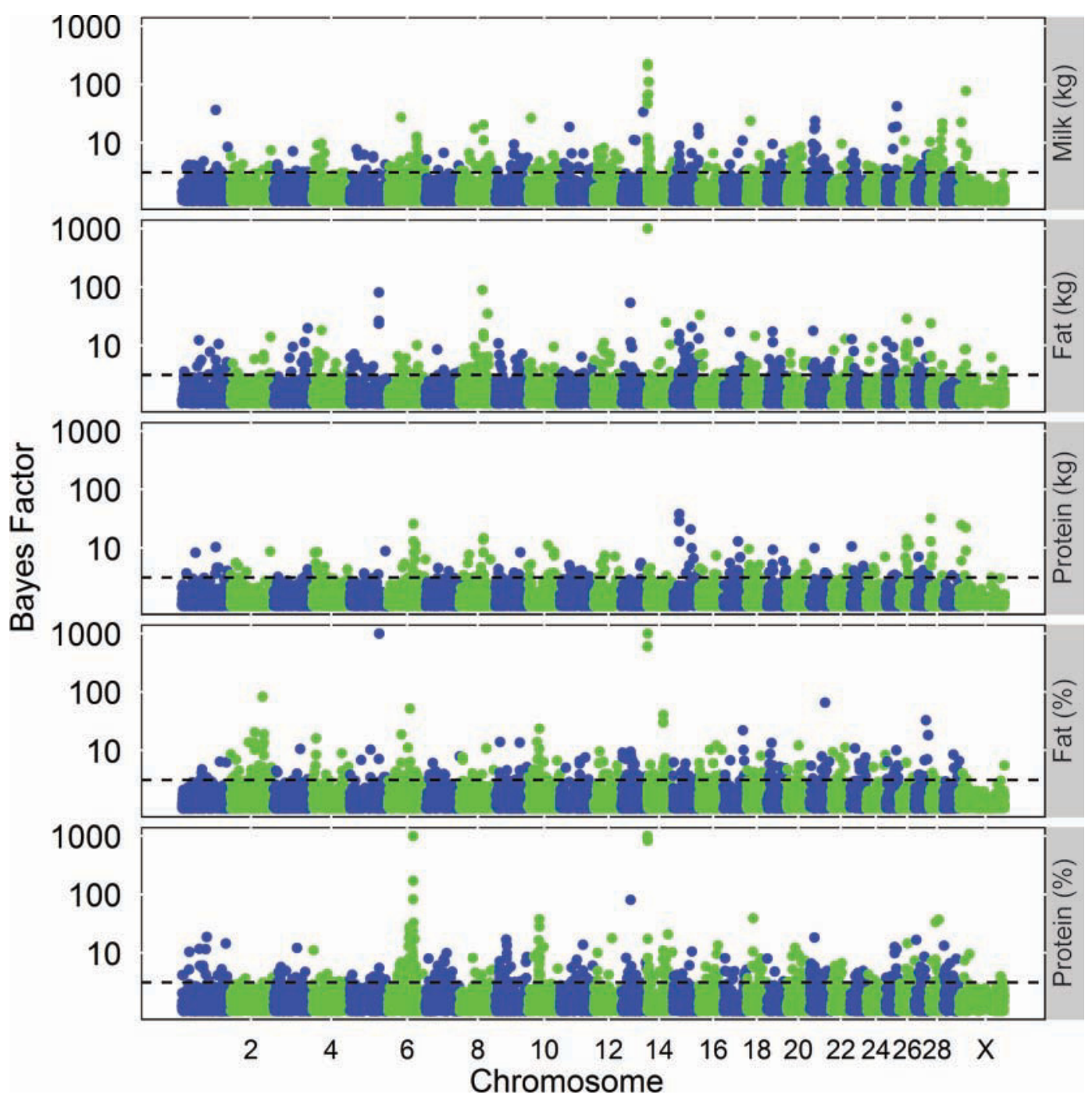

Figure 1. Manhattan plot for each trait with the Bayes Factor (BF) for all individual SNP. The y-axis is presented as a log scale and values with $\mathrm{BF}>1,000$ are presented as $\mathrm{BF}=1,000$; the dashed line corresponds to $\mathrm{BF} 3.1$. Color version available in the online $\mathrm{PDF}$.

correlated trait. The standardized covariance between milk yield and fat yield was 0.52 (Table 5 ). This covariance was mainly explained by SNP in class 0 ; based on these SNP, the covariance was 0.51. This pattern held for the most combinations of traits, except for the correlation between fat yield and fat percentage where the covariance was mainly explained by SNP in class B (0.24 total and 0.16 in class B) and the covariance between fat yield and protein percentage where the total covariance was mainly explained by SNP in class 0 and B (0.01 total, -0.05 in class 0 and 0.06 in class B).

Nine regions were found with SNP having an effect for 2 traits and 1 region on chromosome 14 was assumed to be significant for 4 of the 5 traits. In Table 6 , the standardized variances and covariance per combination of correlated traits for these 10 regions is shown. In this table, the chromosome number, position on the chromosome, and the number of SNP in the regions are shown. For the correlation between protein yield and fat percentage, no significant region was found. Generally, the significant regions had a very small contribution $(<0.01 \%)$ to the standardized SNP variances and covariances of the traits, except for the region found on chromosome 14 (Table 6). This region accounted for $1 \%$ of the SNP variance of milk yield to $41 \%$ of the SNP variance of fat percentage. The standardized covariance of this region for fat yield and fat percentage was 0.16 , whereas the total covariance was 0.24 , so a major part of the covariance was explained by the region on chromosome 14 .

\section{DISCUSSION}

In this study, data were used from 4 experimental herds with different management systems. As estimates for the 
Table 3. Genetic correlations for the production traits calculated traditionally using the polygenic component restricted maximum likelihood (REML; above diagonal) and using SNP (co)variances (below diagonal)

\begin{tabular}{lccccc}
\hline Item & Milk $(\mathrm{kg})$ & Fat $(\mathrm{kg})$ & Protein $(\mathrm{kg})$ & Fat $(\%)$ & Protein $(\%)$ \\
\hline Milk (kg) & & 0.57 & 0.85 & -0.44 & -0.39 \\
Fat (kg) & 0.52 & & 0.79 & 0.48 & 0.31 \\
Protein (kg) & 0.68 & 0.62 & & -0.07 & 0.15 \\
Fat (\%) & -0.23 & 0.24 & -0.10 & & 0.72 \\
Protein (\%) & -0.26 & -0.01 & -0.01 & 0.33 & \\
\hline
\end{tabular}

heritability and genetic correlations in this study were similar to estimates found in other studies (e.g., Misztal et al., 1992; Albuquerque et al., 1995; Miglior et al., 2007; Mucha and Strandberg, 2011), the pre-adjustment of the phenotypic records apparently was adequate. A more detailed analysis of the phenotypic data across the different herds is given by Banos et al. (2011).

\section{Individual SNP Effects}

About 0.5 to $1.0 \%$ of the SNP had a significant effect on one or more traits, ranging from 200,308, and 334 SNP for protein, fat, and milk yield, and 318 and 387 for fat and protein percentage, respectively. Pryce et al. (2010) reported a remarkably similar number

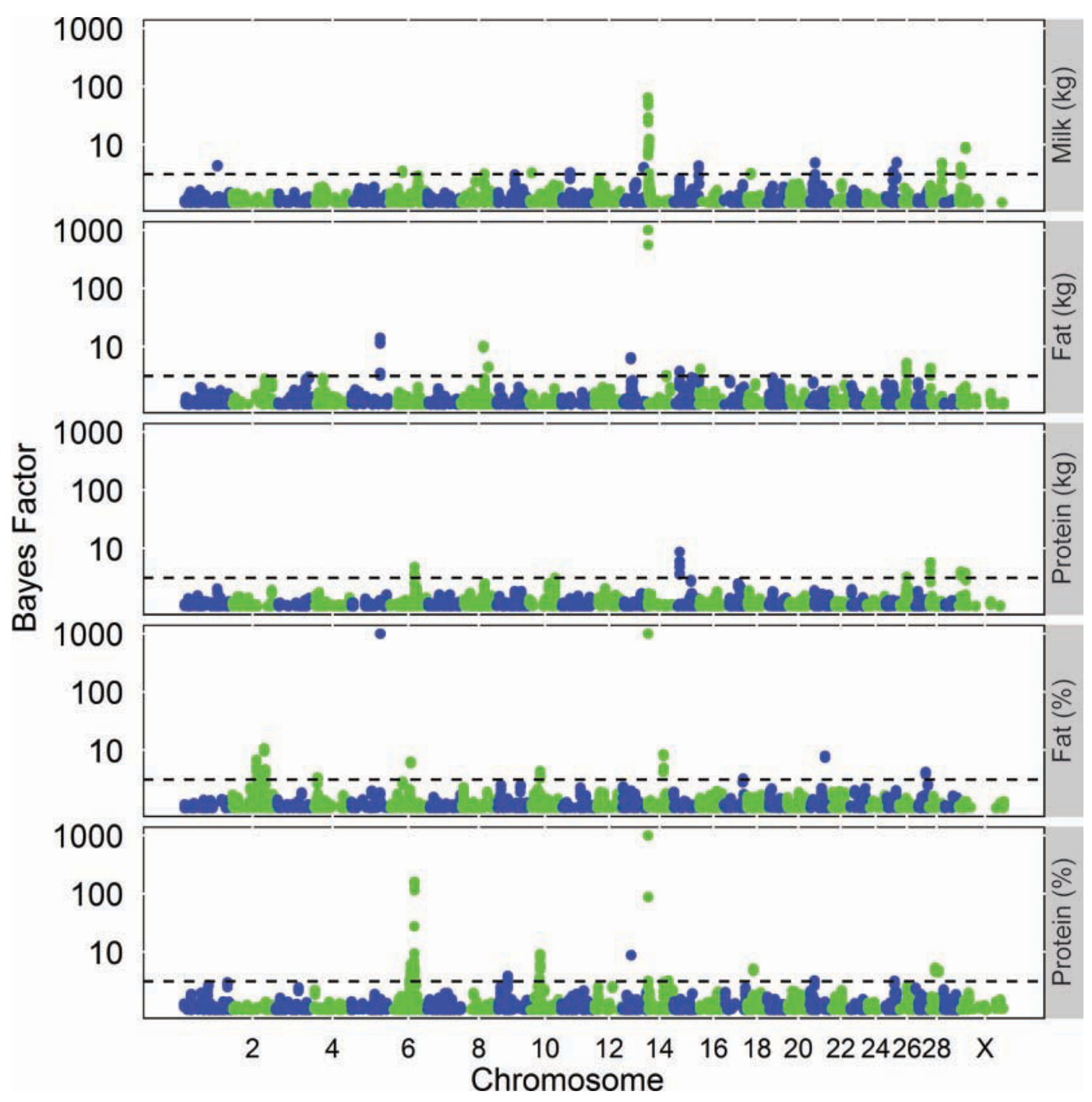

Figure 2. Manhattan plot for each trait with the Bayes Factor (BF) for a sliding window of $10 \mathrm{SNP}$. The y-axis is presented as a log scale and values with $\mathrm{BF}>1,000$ are presented as $\mathrm{BF}=1,000$; the dashed line corresponds to $\mathrm{BF}$ 3.1. Color version available in the online PDF. 
Table 4. Number of SNP with an effect per type of locus class for each pair of correlated traits (total number of SNP $=37,590)^{1}$

\begin{tabular}{|c|c|c|c|c|c|c|c|c|c|c|}
\hline Class $^{2}$ & \multicolumn{10}{|c|}{ Trait 1 ; trait 2} \\
\hline A & 274 & 256 & 251 & 319 & 298 & 196 & 321 & 305 & 195 & 275 \\
\hline B & 58 & 78 & 57 & 0 & 10 & 0 & 0 & 2 & 5 & 43 \\
\hline $\mathrm{C}$ & 2 & 0 & 0 & 15 & 0 & 4 & 13 & 1 & 0 & 0 \\
\hline
\end{tabular}

${ }^{1}$ An SNP was assumed to have an effect if the Bayes Factor (BF) of that SNP was equal to or higher than 3.1.

${ }^{2} 0=$ No SNP with an effect for none of the 2 traits; A = SNP with an effect for trait 1 but no effect for trait $2 ; \mathrm{B}=\mathrm{SNP}$ with an effect in same direction for trait 1 and $2 ; \mathrm{C}=\mathrm{SNP}$ with an effect in opposite directions for trait 1 and $2 ; \mathrm{D}=\mathrm{SNP}$ without an effect for trait 1 but with an effect for trait 2 .

of significant SNP in a Holstein data set using single SNP regressions (i.e., 224, 213, 292, 310, and 461, respectively). When clustering the SNP, Pryce et al. (2010) found a conservative estimate of 304 QTL in the Holstein discovery population for protein percentage. The use of BSSVS in the current study might have had an effect on the results, as SNP effects are sampled from 2 distributions with a small and large variance, and all SNP are fitted simultaneously. Note that in the BSSVS model, many loci receive effects from a distribution with small variance. This may give a somewhat conservative estimate of the number of QTL compared with other genomic prediction models such as BayesB or BayesC. Nevertheless, in previous studies, the ability of BSSVS to detect QTL has been shown repeatedly (Uleberg and Meuwissen, 2007; Veerkamp et al., 2010; Calus et al., 2011b).

Because estimates for individual SNP effects are affected by sampling and mixing of the SNP effects in the BSSVS analysis, we averaged the effects over windows of 10 neighboring SNP and found particular interesting regions on chromosomes $1,13,14,15,21$, 25 , and 28 for milk yield; $5,8,13,15,26$, and 28 for fat yield; $6,15,26$, and 28 for protein yield; $2,5,610$, 14 , and 21 for fat percentage; and $6,10,13,14,18$, and 28 for protein percentage. Ten SNP regions with

Table 5. The standardized SNP variance $\left(\sigma_{1}^{2}\right.$ and $\left.\sigma_{2}^{2}\right)$ and covariance $\left(\sigma_{12}\right)$ per type of loci class and the total standardized covariance of the correlated traits ${ }^{1}$

Trait 1 ; trait 2

Milk $(\mathrm{kg}) ; \quad$ Milk $(\mathrm{kg}) ; \quad$ Fat $(\mathrm{kg}) ; \quad$ Milk $(\mathrm{kg}) ;$ Fat $(\mathrm{kg}) ;$ Protein $(\mathrm{kg}) ;$ Milk $(\mathrm{kg}) ; \quad$ Fat $(\mathrm{kg}) ; \quad$ Protein $(\mathrm{kg}) ; \quad$ Fat $(\%)$; Class $^{2} \quad$ Variance fat $(\mathrm{kg})$ protein $(\mathrm{kg})$ protein $(\mathrm{kg})$ fat $(\%)$ fat $(\%)$ fat $(\%)$ protein $(\%)$ protein $(\%)$ protein $(\%)$ protein $(\%)$

\begin{tabular}{|c|c|c|c|c|c|c|c|c|c|c|c|}
\hline \multirow[t]{3}{*}{0} & $\sigma_{1}^{2}$ & 0.96 & 0.97 & 0.86 & 0.96 & 0.86 & 0.98 & 0.96 & 0.86 & 0.98 & 0.55 \\
\hline & $\sigma_{2}^{2}$ & 0.86 & 0.98 & 0.98 & 0.55 & 0.55 & 0.55 & 0.90 & 0.90 & 0.90 & 0.90 \\
\hline & $\sigma_{12}$ & 0.51 & 0.66 & 0.60 & -0.18 & 0.07 & -0.08 & -0.23 & -0.05 & -0.01 & 0.30 \\
\hline \multirow{2}{*}{ A } & $\sigma_{2}^{2}$ & 0.01 & 0.01 & 0.01 & 0.01 & 0.00 & 0.00 & 0.01 & 0.01 & 0.01 & 0.01 \\
\hline & $\sigma_{12}$ & 0.01 & 0.01 & 0.01 & -0.01 & 0.00 & 0.00 & -0.01 & 0.00 & 0.00 & 0.01 \\
\hline \multirow[t]{2}{*}{ B } & $\sigma_{1}^{2}$ & 0.01 & 0.01 & 0.00 & 0.00 & 0.12 & 0.00 & 0.00 & 0.12 & 0.00 & 0.44 \\
\hline & $\sigma_{12}$ & 0.00 & 0.01 & 0.00 & 0.00 & 0.16 & 0.00 & 0.00 & 0.06 & 0.00 & 0.01 \\
\hline \multirow[t]{3}{*}{$\mathrm{C}$} & $\sigma_{1}^{2}$ & 0.00 & 0.00 & 0.00 & 0.00 & 0.00 & 0.00 & 0.00 & 0.00 & 0.00 & 0.00 \\
\hline & $\sigma_{2}^{2}$ & 0.12 & 0.00 & 0.00 & 0.41 & 0.00 & 0.00 & 0.06 & 0.00 & 0.00 & 0.00 \\
\hline & $\sigma_{12}$ & -0.02 & 0.00 & 0.00 & -0.04 & 0.00 & 0.00 & -0.01 & 0.00 & 0.00 & 0.00 \\
\hline $\mathrm{D}$ & $\sigma_{1}^{2}$ & 0.01 & 0.00 & 0.00 & 0.01 & 0.01 & 0.01 & 0.01 & 0.01 & 0.01 & 0.01 \\
\hline
\end{tabular}

${ }^{1} \mathrm{An}$ SNP was assumed to have an effect if the Bayes Factor (BF) of that SNP was equal to or higher than 3.1.

${ }^{2} 0=$ No SNP with an effect for both traits; $\mathrm{A}=\mathrm{SNP}$ with an effect for trait 1 but no effect for trait $2 ; \mathrm{B}=\mathrm{SNP}$ with an effect in same direction for trait 1 and 2; C = SNP with an effect in opposite directions for trait 1 and $2 ; \mathrm{D}=\mathrm{SNP}$ without an effect for trait 1 but with an effect for trait 2 . 
Table 6. Chromosome number, number of SNP, position on the chromosome of the first and last SNP, affected traits, standardized SNP variance $\left(\sigma_{1}^{2}\right.$ and $\left.\sigma_{2}^{2}\right)$ and covariance $\left(\sigma_{12}\right)$ per SNP region ${ }^{1}$

\begin{tabular}{|c|c|c|c|c|c|c|c|c|}
\hline Chromosome & No. of SNP & \multicolumn{2}{|c|}{ Position on chromosome } & Trait 1 & Trait 2 & $\sigma_{1}^{2}$ & $\sigma_{2}^{2}$ & $\sigma_{12}$ \\
\hline 5 & 3 & 94858411 & 95006068 & Fat $(\mathrm{kg})$ & Fat $(\%)$ & 0.000 & 0.000 & 0.000 \\
\hline 6 & 10 & 82078166 & 82605943 & Protein (kg) & Protein (\%) & 0.000 & 0.000 & 0.000 \\
\hline 10 & 10 & 36978160 & 37594262 & Fat $(\%)$ & Protein (\%) & 0.000 & 0.001 & 0.000 \\
\hline \multirow[t]{3}{*}{14} & 11 & 1463676 & 2117455 & Milk (kg) & Fat $(\mathrm{kg})$ & 0.008 & 0.117 & -0.019 \\
\hline & & & & Fat $(\mathrm{kg})$ & Protein (\%) & 0.117 & 0.059 & 0.058 \\
\hline & & & & Fat $(\%)$ & Protein (\%) & 0.414 & 0.059 & 0.005 \\
\hline 15 & 4 & 21162454 & 21283666 & Fat $(\mathrm{kg})$ & Protein (kg) & 0.000 & 0.000 & 0.000 \\
\hline 26 & 5 & 25032529 & 25314352 & Fat (kg) & Protein (kg) & 0.000 & 0.000 & 0.000 \\
\hline 28 & 10 & 4408535 & 5052476 & Fat (kg) & Protein (kg) & 0.000 & 0.001 & 0.001 \\
\hline $\mathrm{X}$ & 9 & 7042383 & 7425524 & Milk (kg) & Protein (kg) & 0.000 & 0.000 & 0.000 \\
\hline
\end{tabular}

${ }^{1}$ An SNP region was assumed to have an effect if the Bayes Factor (BF) of a window of 10 SNP was equal to or higher as 3.1 for both traits.

significant effects affected 2 or more traits (Table 6). The regions on chromosome 5 contained 3 SNP that had an effect on both fat percentage and fat yield. In the review of Khatkar et al. (2004), few studies were reported with QTL for either of these traits in this region, but Pryce et al. (2010) also reported significant QTL for fat yield on chromosome 5. Many significant QTL were reported on chromosome 6, some of which were attributed to the casein gene complex (Khatkar et al., 2004; Ogorevc et al., 2009). We found 2 regions on chromosome 6, 1 affecting fat and protein percentage and 1 affecting protein yield and protein percentage, which agreed with the 2 regions reported by Schrooten et al. (2004). In the SNP region found on chromosome 14, other studies (e.g., Grisart et al., 2002; Spelman et al., 2002) identified a gene with a major effect on milk production traits, the DGAT1 gene, which also explains a large part of the variance for fat percentage in genomic prediction models (de Roos et al., 2007). For the other chromosomes with a region affecting more than 1 trait $(10,15,26$, and 28) several QTL were reported affecting milk traits (Khatkar et al., 2004; Ogorevc et al., 2009). In conclusion, the regions that showed high posterior QTL probabilities were in good agreement with regions for which QTL were reported in the literature. However, the objective of this study was not to identify functional QTL, but to investigate the makeup of the genetic (co)variances between milk yield, fat yield, protein yield, and their percentages.

\section{Variances Explained by SNP}

The performed genome-wide association study fitted all SNP simultaneously using BSSVS and all significant
SNP explained $0,14,2,45$, and $10 \%$ of the genetic variance in milk, fat, and protein yield, and fat and protein percentage, respectively. The heritability for these significant SNP was 0 for milk and protein yield; for fat yield, fat percentage, and protein percentage, these values were $0.05,0.20$, and 0.04 , respectively. When excluding results from the DGAT1 region, the many significant SNP and regions explained even less genetic variance. This is consistent with the results presented by Hayes et al. (2010), who report differences in the genetic architecture of traits investigated, where, in particular, fat percentage had 1 locus of large effect (DGAT1 region) and many loci with very small effects, but cow overall conformation (overall type) only showed loci of small effect. Still, using both significant and nonsignificant SNP, we obtained estimates for the variances very close to the estimates obtained with REML, albeit some different portioning was observed between the traits.

The significance level used (BF) will affect the number of QTL declared significant, and this might, in turn, affect the makeup of covariances. Therefore, to investigate the effects of a too stringent testing criteria on the variances and number of SNP in the different classes of Bohren et al. (1966), we repeated the analysis with a higher false discovery rate by selecting all SNP that had a posterior probability higher than the prior probability (i.e., a $\mathrm{BF}>1$ ). Thereby, the proportion of significant SNP increased from 0.5 to $1.0 \%$ to $37 \%$. However, even with this high false discovery rate, still between 52 and $63 \%$ of the genetic variances was explained by SNP in class 0, apart from fat percentage where $34 \%$ was in class 0. Thus, the importance of nonsignificant SNP remains, despite less stringent testing. 
The first reason why we might have missed QTL is that the remaining QTL for the yield traits just have a small effect. A second reason why we might have missed QTL is that some QTL are not in perfect linkage disequilibrium with the SNP used here. Although the distance between SNP might have been close enough to capture most of the genetic variation (e.g., de Roos et al., 2008), differences in minor allele frequency between the SNP on the chip and the minor allele frequency of the QTL might have hindered identifying significant QTL with low minor allele frequency. In human populations, where linkage disequilibrium is generally lower than in the cattle population, this effect of different minor allele frequency between QTL and SNP is likely to explain a considerable part of the missing heritability, even with many more SNP included in the analysis (Visscher et al., 2010; Yang et al., 2010).

Hence, it appears surprising that still considerable genetic variance is explained by BSSVS, given the small number of significant QTL identified. A likely explanation for why SNP with a small effect explain so much variance is that these SNP pick up the relationships between animals, including Mendelian sampling, This is in contrast to studies in humans where part of the heritability was missing, because unrelated individuals were used (Visscher et al., 2010; Yang et al., 2010). This admixture in our data due to relationships between animals was picked up by the SNP, and made up for the genetic variance not picked up by significant QTL, even though, by fitting the polygenic effect, some adjustment was made for the average relationships between animals.

\section{Makeup of the Genetic Covariance}

As expected, with stronger genetic correlations between 2 traits, more SNP were found that affected both traits. For example, between milk and protein yield, the SNP covariance was 0.68 and $23 \%(78 / 334)$ and $39 \%$ $(78 / 200)$ of the significant SNP also affected the other trait, all in the same direction. With a lower genetic correlation between 2 traits, a lower number of SNP also were found to affect both traits (e.g., up to 3 SNP affecting fat kilograms and protein percentage). All 3 SNP that affected 2 traits showed the same direction of effect (i.e., only 3 SNP were identified breaking the genetic correlations). Investigating the makeup of the genetic correlations at the QTL level needs more certainty about the location of QTL and the direction and size of the allele substitution effects. This certainty can be gained by validating the QTL in validation data sets or by proper significance testing and accounting for false discovery rate (Schrooten et al., 2004; Bagnato et al., 2008; Chamberlain et al., 2008; Lipkin et al., 2008b;
Bolormaa et al., 2010). We tested the adjustments suggested by Chamberlain et al. (2008), which resulted in more SNP being allocated in class $0, \mathrm{~B}$, and $\mathrm{C}$, and no SNP in the classes affecting 1 trait (class A and D). This might have been because of using too stringent testing criteria in the first place and the assumption that we might have found all QTL. Another cause of uncertainty in the QTL might be environmental covariances between traits. Environmental covariance between traits might lead to the same SNP being significant for 2 traits, whereas no pleiotropic effect exists (for example, because fat yield and protein yield both rely on the volume of milk). Therefore, to investigate the different genetic pathways, it might be more appropriate to make traits independent by adjusting them to each other.

Here, we investigated the makeup of the genetic correlation at the level of variance explained, by comparing the genetic correlations estimated with the polygenic model in ASReml and the SNP (co)variances of BSSVS (Table 3). For some combination of traits important differences existed. For example the genetic correlation between fat percentage and protein percentage was 0.72 , whereas the covariance explained by the SNP was 0.33 only. Also, between fat yield and protein percentage, no genetic covariance was detected using the individual SNP, whereas the genetic correlation from the polygenic model was 0.31 . Also, for protein percentage, the SNP correlation was 0 with protein yield, whereas the correlations from the polygenic ASReml model where positive. Hence, clear differences exist between the polygenic and SNP correlations and these differences are difficult to pinpoint at individual QTL. An exception was when SNP were grouped in regions (Table 6); then, the standardized covariance between fat yield and protein percentage of the DGAT1 region was 0.058. Still, it remains difficult to identify the differences between SNP and polygenic covariances.

Some improvements of the BSSVS model might facilitate this work further. For example, sampling SNP for the combined effect for all traits simultaneously might have identified individual SNP affecting the 5 traits more accurately, as demonstrated recently (Calus et al., 2011b; Calus and Veerkamp, 2011). In comparison with the univariate model used here, in a bivariate analysis it is also possible to model the polygenic covariances between traits. In comparison with the univariate model used here, this would have given a more complete parameterization and probably a more accurate partitioning of the genetic covariance due to SNP and polygenic components (Calus and Veerkamp, 2011). However, the relatively early stages of development of the multipletrait BSSVS at the start of the study and the larger number of bivariate analyses required led us to use the 
univariate approach in this study. Ideally, the results should be replicated in the future with a multiple-trait BSSVS including all 5 traits simultaneously. Also, to study the genetic makeup of the covariance, it might be worthwhile to extend the BSSVS model to sample $\pi$ (the proportion of loci with small effect), adopt a model where a proportion $\pi$ of the loci have 0 instead of small effects (Habier et al., 2011), or extend the BSSVS model to sample the ratio between the variance of the large and small QTL effects. This would enable a more direct learning of the model from the data about the number of QTL underlying the genetic (co)variances.

\section{Asymmetry of Genetic Response}

The DGAT1 region explained a relatively large part of the variance $(>40 \%)$ for fat percentage and a large part of the variances and covariances of milk yield, fat yield, and protein percentage. The correlation between fat yield and fat percentage, which was mainly (0.16 of 0.24 ) explained by this gene, is expected to be much smaller after fixation of the DGAT1 gene, partly through its direct effect on the genetic covariance as estimated here, but also because of its larger contribution to the genetic variation of fat percentage. Another factor that affected the correlations between the traits in this study was that fat yield and fat percentage are ratios of each other. For ratio traits, it is the relative values of the coefficient of variation for milk and fat yield that determine the genetic correlations between fat yield and fat percentage (Simm et al., 1987; Lipkin et al., 2008a). However, here we focused on the simplest condition for asymmetry of correlated responses, which is the presence of type $\mathrm{C}$ loci (i.e., affecting 2 traits in a different direction) with allele frequencies of 0.2 or 0.8 (Bohren et al., 1966). According to the present study, 2 SNP with a very high BF were found in the $D G A T 1$ region for fat yield and fat percentage. These SNP had both an allele frequency around 0.3 and were present in class B. The allocation to class B and C is somewhat arbitrary and depends on the orientation of the selection. Consider a scenario where selection aims to decrease fat percentage, but to increase fat yield (for example, when a quota on fat percentage exists; Veerkamp et al., 2002). For such a breeding goal, the DGAT1 region will have a high contribution to asymmetry in correlated response between fat yield and fat percentage. No other regions were found with such a major effect on the variances and covariances of the different traits that asymmetry of genetic correlations was expected. Because estimated covariances using the polygenic and BSSVS model were very similar, even for traits heavily affected by DGAT1, the infinitesimal model is expected to be sufficient for the estimation of the variances and covariances between milk production traits.

\section{CONCLUSIONS}

As correlated responses between traits may differ, depending on the makeup of genetic covariances, and may differ from the predictions of polygenic covariances, we investigated the makeup of the genetic covariances between milk production traits using high-density genotypes. Genetic correlations based on SNP or pedigree information differed and few SNP that had a substantial effect on one or more traits were found. The SNP with no significant effect explained most of the SNP variances and covariances. Therefore, little asymmetry of selection is expected, and SNP genetic correlations approximate the polygenic correlations usually used to predict correlated responses. The exception is the DGAT1 region, which explained 1 to $41 \%$ of the variances of 4 traits and explained a major part of the positive correlation between fat yield and fat percentage. It is expected that this region may contribute to asymmetry in correlated response between fat yield and fat percentage if selection for these 2 traits is in the opposite direction.

\section{ACKNOWLEDGMENTS}

The RobustMilk project is thanked for providing the data, in particular Donagh Berry (Teagasc Moorepark, Fermoy, Co. Cork, Ireland), Eileen Wall [Scottish Agricultural College (SAC), Edinburgh, UK], and Erling Strandberg [Swedish University of Agricultural Sciences (SLU), Uppsala, Sweden]. The RobustMilk project (http://www.robustmilk.eu) is financially supported by the European Commission under the Seventh Research Framework Program, Grant Agreement KBBE-211708. The content of this paper is the sole responsibility of the authors, and it does not necessarily represent the views of the Commission or its services.

\section{REFERENCES}

Albuquerque, L. G., G. Dimov, J. F. Keown, and L. D. Van Vleck. 1995. Estimates using an animal model of (co)variances for yields of milk, fat, and protein for the first lactation of Holstein cows in California and New York. J. Dairy Sci. 78:1591-1596.

Bagnato, A., F. Schiavini, A. Rossoni, C. Maltecca, M. Dolezal, S. I. Medugorac, J. Sölkner, V. Russo, L. Fontanesi, A. Friedmann, M. Soller, and E. Lipkin. 2008. Quantitative trait loci affecting milk yield and protein percentage in a three-country Brown Swiss population. J. Dairy Sci. 91:767-783.

Banos, G., M. P. Coffey, R. F. Veerkamp, D. P. Berry, and E. Wall 2011. Merging and characterising phenotypic data on conventional and rare traits from dairy cattle experimental resources in three countries. Animal http://dx.doi.org/10.1017/S1751731111002655.

Bohren, B. B., W. G. Hill, and A. Robertson. 1966. Some observations on asymmetrical correlated responses to selection. Genet. Res. 7:44-57 
Bolormaa, S., J. E. Pryce, B. J. Hayes, and M. E. Goddard. 2010. Multivariate analysis of a genome-wide association study in dairy cattle. J. Dairy Sci. 93:3818-3833.

Browning, S. R., and B. L. Browning. 2007. Rapid and accurate haplotype phasing and missing-data inference for whole-genome association studies by use of localized haplotype clustering. Am. J. Hum. Genet. 81:1084-1097.

Calus, M. P. L., T. H. E. Meuwissen, A. P. W. de Roos, and R. F. Veerkamp. 2008. Accuracy of genomic selection using different methods to define haplotypes. Genetics 178:553-561.

Calus, M. P. L., H. A. Mulder, and J. W. M. Bastiaansen. 2011a. Identification of Mendelian inconsistencies between SNP and pedigree information of sibs. Genet. Sel. Evol. 43:34.

Calus, M. P. L., H. A. Mulder, and R. F. Veerkamp. 2011b. Estimating genomic breeding values and detecting QTL using univariate and bivariate models. BMC Proc. 5(Suppl. 3):S5.

Calus, M. P. L., and R. F. Veerkamp. 2011. Accuracy of multi-trait genomic selection using different methods. Genet. Sel. Evol. 43:26.

Chamberlain, A. J., H. C. McPartlan, and M. E. Goddard. 2008. Genes influencing milk production traits predominantly affect one of four biological pathways. Genet. Sel. Evol. 40:79-89.

de Roos, A. P. W., B. J. Hayes, R. J. Spelman, and M. E. Goddard. 2008. Linkage disequilibrium and persistence of phase in HolsteinFriesian, Jersey and Angus cattle. Genetics 179:1503-1512.

de Roos, A. P. W., C. Schrooten, E. Mullaart, M. P. L. Calus, and R. F. Veerkamp. 2007. Breeding value estimation for fat percentage using dense markers on Bos taurus autosome 14. J. Dairy Sci. 90:4821-4829.

Gardner, K. M., and R. G. Latta. 2007. Shared quantitative trait loci underlying the genetic correlation between continuous traits. Mol. Ecol. 16:4195-4209.

Gilmour, A. R., B. R. Cullis, S. J. Welham, and R. Thompson. 2009. ASREML. Program User Manual. NSW Agriculture, Orange Agricultural Institute, Orange NSW, Australia.

Grisart, B., W. Coppieters, F. Farnir, L. Karim, C. Ford, P. Berzi, N. Cambisano, M. Mni, S. Reid, P. Simon, R. Spelman, M. Georges, and R. Snell. 2002. Positional candidate cloning of a QTL in dairy cattle: Identification of a missense mutation in the bovine DGAT1 gene with major effect on milk yield and composition. Genome Res. 12:222-231.

Habier, D., R. L. Fernando, K. Kizilkaya, and D. J. Garrick. 2011. Extension of the Bayesian alphabet for genomic selection. BMC Bioinformatics 12:186.

Hayes, B. J., J. Pryce, A. J. Chamberlain, P. J. Bowman, and M. E. Goddard. 2010. Genetic architecture of complex traits and accuracy of genomic prediction: Coat colour, milk-fat percentage, and type in Holstein cattle as contrasting model traits. PLoS Genet. 6:e1001139.

Kass, R. E., and A. E. Raftery. 1995. Bayes factors. J. Am. Stat. Assoc. 90:773-795.

Khatkar, M. S., P. C. Thomson, I. Tammen, and H. W. Raadsma. 2004. Quantitative trait loci mapping in dairy cattle: Review and meta-analysis. Genet. Sel. Evol. 36:163-190.

Lipkin, E., A. Bagnato, and M. Soller. 2008a. Expected effects on protein yield of marker-assisted selection at quantitative trait loci affecting milk yield and milk protein percentage. J. Dairy Sci. 91:2857-2863.

Lipkin, E., R. Tal-Stein, A. Friedmann, and M. Soller. 2008b. Effect of quantitative trait loci for milk protein percentage on milk protein yield and milk yield in Israeli Holstein dairy cattle. J. Dairy Sci. 91:1614-1627.

McMillan, I., R. W. Fairfull, G. W. Friars, and M. Quinton. 1995. The effect of simultaneous selection on the genetic correlation. Theor. Appl. Genet. 91:776-779.

Meuwissen, T. H. E., and M. E. Goddard. 2004. Mapping multiple QTL using linkage disequilibrium and linkage analysis information and multitrait data. Genet. Sel. Evol. 36:261-279.
Miglior, F., A. Sewalem, J. Jamrozik, J. Bohmanova, D. M. Lefebvre, and R. K. Moore. 2007. Genetic analysis of milk urea nitrogen and lactose and their relationships with other production traits in Canadian Holstein cattle. J. Dairy Sci. 90:2468-2479.

Misztal, I., T. J. Lawlor, T. H. Short, and P. M. VanRaden. 1992. Multiple-trait estimation of variance components of yield and type traits using an animal model. J. Dairy Sci. 75:544-551.

Mucha, S., and E. Strandberg. 2011. Genetic analysis of milk urea nitrogen and relationships with yield and fertility across lactation. J. Dairy Sci. 94:5665-5672.

Ogorevc, J., T. Kunej, A. Razpet, and P. Dovc. 2009. Database of cattle candidate genes and genetic markers for milk production and mastitis. Anim. Genet. 40:832-851.

Parker, R. J., L. D. McGilliard, and J. L. Gill. 1970. Genetic correlation and response to selection in simulated populations. III. Correlated response to selection. Theor. Appl. Genet. 40:157-162.

Pryce, J. E., S. Bolormaa, A. J. Chamberlain, P. J. Bowman, K. Savin, M. E. Goddard, and B. J. Hayes. 2010. A validated genome-wide association study in 2 dairy cattle breeds for milk production and fertility traits using variable length haplotypes. J. Dairy Sci. 93:3331-3345.

Quinton, M., and I. McMillan. 1996. The effect of index selection on allele frequencies and future genetic gains when traits are correlated. Theor. Appl. Genet. 93:1335-1342.

Rauw, W. M., E. Kanis, E. N. Noordhuizen Stassen, and F. J. Grommers. 1998. Undesirable side effects of selection for high production efficiency in farm animals: A review. Livest. Prod. Sci. 56:15-33.

Schrooten, C., M. C. A. M. Bink, and H. Bovenhuis. 2004. Whole genome scan to detect chromosomal regions affecting multiple traits in dairy cattle. J. Dairy Sci. 87:3550-3560.

Simm, G., C. Smith, and R. Thompson. 1987. The use of product traits such as lean growth rate as selection criteria in animal breeding. Anim. Prod. 45:307-316.

Spelman, R. J., C. A. Ford, P. McElhinney, G. C. Gregory, and R. G. Snell. 2002. Characterization of the DGAT1 gene in the New Zealand dairy population. J. Dairy Sci. 85:3514-3517.

Uleberg, E., and T. H. E. Meuwissen. 2007. Fine mapping of multiple QTL using combined linkage and linkage disequilibrium mapping - A comparison of single QTL and multi QTL methods. Genet. Sel. Evol. 39:285-299.

Veerkamp, R. F., K. L. Verbyla, H. A. Mulder, and M. P. L. Calus. 2010. Simultaneous QTL detection and genomic breeding value estimation using high density SNP chips. BMC Proc. 4:S9.

Veerkamp, R. F., B. Beerda, and T. van der Lende. 2003. Effects of genetic selection for milk yield on energy balance, levels of hormones, and metabolites in lactating cattle, and possible links to reduced fertility. Livest. Prod. Sci. 83:257-275.

Veerkamp, R. F., P. Dillon, E. Kelly, A. R. Cromie, and A. F. Groen. 2002. Dairy cattle breeding objectives combining yield, survival and calving interval for pasture-based systems in Ireland under different milk quota scenarios. Livest. Prod. Sci. 76:137-151.

Verbyla, K. L., M. P. L. Calus, H. A. Mulder, Y. de Haas, and R. F. Veerkamp. 2010. Predicting energy balance for dairy cows using high density SNP information. J. Dairy Sci. 93:2757-2764.

Visscher, P. M., and R. Thompson. 1992. Univariate and multivariate parameter estimates for milk production traits using an animal model. I. Description and results of REML analyses. Genet. Sel. Evol. 24:415-430.

Visscher, P. M., J. A. Yang, and M. E. Goddard. 2010. A commentary on 'Common SNPs explain a large proportion of the heritability for human height' by Yang et al. (2010). Twin Res. Hum. Genet. 13:517-524.

Yang, J., B. Benyamin, B. P. McEvoy, S. Gordon, A. K. Henders, D. R. Nyholt, P. A. Madden, A. C. Heath, N. G. Martin, G. W. Montgomery, M. E. Goddard, and P. M. Visscher. 2010. Common SNPs explain a large proportion of the heritability for human height. Nat. Genet. 42:565-569. 